\title{
INFORME: AVANCES Y RETROCESOS DE LA SEGURIDAD SOCIAL EN MÉXICO, 2018
}

\author{
REPORT: PROGRESS AND SETBACKS OF SOCIAL \\ SECURITY IN MEXICO, 2018
}

\author{
RAPPORT: PROGRES ET RECULS DE LA SÉCURITÉ \\ SOCIALE DU MEXIQUE, 2018
}

\author{
Gabriela MENDIZÁBAL BERMÚDEZ*
}

\section{INTRODUCCIÓN}

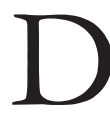

urante 2018 se presentaron cambios importantes en el país. Dentro de ellos se pueden resaltar las consecuencias del sismo de 2017; el éxodo de migrantes centroamericanos; la transición presidencial y sus consecuentes proyectos legislativos; las pérdidas de las Afores y la eminente falta de reformas legislativas en seguridad social.

En ese orden de ideas, las prioridades marcadas en materia de seguridad social partieron en la atención a la contingencia generada por los sismos, y dieron como respuesta la estrategia denominada "Fuerza México", integrada de varios elementos: la entrega inmediata de víveres, limpieza y remoción de escombros, y, finalmente, la reconstrucción de viviendas; sin embargo, con el cambio de administración aquélla fue sustituida por el Plan Nacional de Reconstrucción, que comenzó a operar a partir del 11 de diciembre de 2018.

Un segundo tema es la expectativa que han generado las denominadas "caravanas migrantes", puesto que durante 2018 sumaron alrededor de 11,5001 migrantes provenientes principalmente de Honduras y El Salvador, quienes

* Profesora investigadora, titular C, de la Facultad de Derecho y Ciencias Sociales en la Universidad Autónoma del Estado de Morelos.

1 "No es una caravana (de migrantes), es un éxodo: las razones de los cientos de hondureños que siguen los pasos de los que ya caminan por México", BBC, Guatemala, 2018, disponible 
rechazaron el plan "Estás en Casa", que les ofrecía atención médica, escuela para los niños y niñas, contar con una identificación oficial para realizar trámites y acceder al Programa de Empleo Temporal, ${ }^{2}$ ya que los migrantes sostienen que no planean quedarse en México. No obstante lo anterior, aún subsiste la incertidumbre de la atención que se les deberá brindar en caso de que se establezcan de forma definitiva en el país, pues no bastará con la asistencia social que hasta el momento se les ha ofrecido.

En tercer lugar, otro suceso importante para el derecho social fue la reforma integral que se generó a partir de la entrada en vigor de la Ley Federal de Declaración Especial de Ausencia para Personas Desaparecidas, que incluyó modificaciones a la Ley Federal del Trabajo, a la Ley del Seguro Social y a la Ley del Instituto de Seguridad y Servicios Sociales, atribuyendo consecuencias a la desaparición de un trabajador similares a las de los riesgos de trabajo, con relación a los derechos a pensiones y recisión laboral.

Además de lo anterior, un hecho alarmante es la pérdida que han tenido los Fondos de Ahorro para el Retiro, siendo la más grande registrada en octubre, que sumó 3 billones 284,428 millones de pesos ${ }^{3}$ (172,592,117.7 dólares) ${ }^{4}$ en cuatro de las principales Administradoras de Fondos para el Retiro (Afores) del país: Inbursa, PensionISSSTE, Profuturo y Banorte.

Por último, se debe resaltar que el segundo semestre del año estuvo marcado, sin lugar a dudas, por la elección presidencial, así como de diputados y senadores, considerada como la elección más grande de la historia de México, donde ganó el partido de izquierda: Movimiento de Regeneración Nacional (Morena), liderada por Andrés Manuel López Obrador, quien con un 53.17\% del total de los votos obtuvo la presidencia de México. ${ }^{5}$ El Congreso de la Unión cambió su conformación radicalmente con 55 escaños de los 128 que

en: https://mmw.bbc.com/mundo/noticias-america-latina-45978111, fecha de consulta: 21 de marzo de 2019.

2 "Migrantes en asamblea rechazan el plan de Peña Nieto", El Universal, disponible en: https:// www.eluniversal.com.mx/nacion/politica/ migrantes-en-asamblea-rechazan-plan-de-pena-nieto (fecha de consulta: 9 de enero de 2018).

3 El Economista, "Minusvalías por 131,000 mdp en afores, tras cancelación del NAIM", disponible en: bttps://mmw.eleconomista.com.mx/sectorfinanciero/Minusvalias-por-131000-mdp-en-afo res-tras-cancela cion-del-NAIM-20181105-0137.html (fecha de consulta: 9 de enero de 2019).

4 El tipo de cambio utilizado para el presente informe es 19.03 pesos mexicanos $=1$ dólares, información del Banco de México al 28 de enero de 2019, Tipo de cambio, Banco de México, disponible en: http:// wmw.anterior.banxico.org.mx/portal-mercado-cambiario/index.html, fecha de consulta 28 de enero de 2019.

5 “INE, López Obrador gana con 30 millones de votos", Proceso, dosponible en: https:// 
conforman el Senado provenientes del mismo partido político del presidente. El caso de la Cámara de Diputados es similar: 256 diputados de los 500 representantes del mismo partido. Además, hubo cambio a inicios de 2019, también de la presidencia de la Suprema Corte de Justicia, encabezada por el ministro Luis María Aguilar Morales. ${ }^{6}$

Los principales cambios que se dieron en el último mes del año fueron la eliminación de las controvertidas pensiones de los expresidentes; la firma del acuerdo mediante el cual se pretende sustituir el Seguro Popular; la iniciativa de reforma laboral integral; la puesta en marcha del programa "Jóvenes Construyendo un Futuro", y, finalmente, la reducción del gasto en el presupuesto de egresos de 2019 para las estancias infantiles, personas con discapacidad, y desaparece el programa denominado "Modelo de atención integral para pacientes con cáncer cervicouterino localmente avanzado" (Micaela).

De tal forma que los sucesos, hechos y cambios normativos son abordados en este informe de la siguiente manera: introducción, marco sociodemográfico y político, los cambios en programas sociales, las resoluciones de la Corte más importantes, las reformas y expectativas legislativas, las conclusiones, y, finalmente, las fuentes de investigación. Desarrollamos cada apartado con ayuda del método analítico, con el objetivo de brindar un panorama general en temas relacionados con la seguridad social en México en 2018.

\section{CONTEXTO SOCIOECONÓMICO Y POLÍTICO}

El 2018 fue un año — que, como ya se adelantó en la introducción— tuvo muchos cambios en materia política, económica y social. Las tensiones políticas fueron un factor importante, pues al ser un año electoral donde se eligió a través de votación popular al titular del Ejecutivo federal y a los titulares del Poder Legislativo, éstas se reflejaron en diversas materias, y la seguridad social no se escapó a dicha influencia.

Los datos sociodemográficos a resaltar para 2018 en México son los siguientes:

www.proceso.com.mx/541748/ine-lopez-obrador-gana-con-30-millones-de-votos-y-el-53-17-por-ciento (fecha de consulta: 9 de enero de 2019).

6 Fue nombrado el 2 de enero de 2019 por un periodo de cuatro años en el cargo de presidente de la Suprema Corte de Justicia de la Nación, Presidencia, SCJN, México, 2019, disponible en: https://wmw.scin.gob.mx/presidencia (fecha de consulta: 5 de febrero de 2019). 
En materia de economía, y según datos reportados por el Banco de México, el Producto Interno Bruto fue del 3.5\% en las actividades primarias del $2.3 \%$, y de las secundarias fue del 1.2\%. La inflación acumulada fue de 4.83 (casi dos puntos menos que la de 2017). ${ }^{7}$ Nuevamente continuó el crecimiento de las remesas provenientes de Estados Unidos. El Banco de México informó que en septiembre ingresaron al país 33,480 millones de dólares americanos por concepto de remesas familiares, lo que representa un crecimiento del $10.53 \%$ en comparación al año anterior. El crecimiento de las remesas acumuló treinta meses consecutivos, sin duda, un hecho histórico en la vida económica de México. ${ }^{8}$

En materia de empleo, en el tercer trimestre de 2018, conforme a la Encuesta Nacional de Ocupación y Empleo (ENOE) presentada por el Instituto Nacional de Estadística y Geografía (INEGI), la población económicamente activa (PEA) fue de 54.3 millones de personas; la cual se compone tanto por la población desocupada, ${ }^{9}$ que comprende a 1.9 millones de personas, y por la población ocupada, ${ }^{10}$ con 52.4 millones de personas. ${ }^{11}$

7 Inflación, Banco de México, disponible en: http://wmw.anterior.banxico.org.mx/portal-infla cion/inflacion.html (fecha de consulta: 24 de enero de 2019).

8 Remesas imparables, bilan 30 meses con crecimiento, $+8.8 \%$ en septiembre, México, BBVA Research, 2018, disponible en: https://wnw.bbvaresearch.com/wp-content/ uploads/2018/11/20181101_Flash Migracion.pdf (fecha de consulta 26 de enero de 2019).

9 Se refiere al porcentaje de la población económicamente activa (PEA) que no trabajó siquiera una hora durante la semana de referencia de la encuesta, pero manifestó su disposición para hacerlo e hizo alguna actividad por obtener empleo, Glosario, INEGI, disponible en bttp:// www.beta.inegi.org. $m x /$ app/glosario/default.html? $p=E N E U$ (fecha de consulta: 22 de marzo de 2019).

10 Comprende las personas de doce y más años de ambos sexos que durante la semana de referencia presentaron una de las siguientes situaciones: trabajaron al menos una hora o un día para producir bienes y servicios a cambio de una remuneración monetaria o en especie; tenían empleo, pero no trabajaron por alguna causa sin dejar de percibir su ingreso; tenían empleo, pero no trabajaron por alguna causa, dejando de percibir su ingreso pero con retorno asegurado a su trabajo en menos de cuatro semanas; no tenían empleo, pero iniciarán con seguridad uno en cuatro semanas o menos; trabajaron al menos una hora o un día en la semana de referencia, sin recibir pago alguno (ni monetario ni en especie) en un negocio propiedad de un familiar o no familiar. Idem. Con ello se contabiliza como ocupadas a las personas que trabajan en la informalidad.

11 Resultados de la Encuesta Nacional de Ocupación y Empleo, INEGI, México, 2018, disponible en: http:// wmw.beta.inegi.org. $m x /$ app/saladeprensa/noticia.html?id $=4558$ (fecha de consulta 27 de enero de 2019). 
Dentro del sector informal, para este periodo se contabilizaron $30.6 \mathrm{mi}$ llones de personas, ${ }^{12}$ lo que representa el $56.7 \%$ de la población ocupada, así como un aumento del $2.2 \%$ respecto del mismo mes del año 2017.

El salario mínimo subió un 3.9\% respecto al año anterior, ya que pasó de 80.4 pesos (4.22 dólares) a 88.36 (4.64 dólares) para 2018.

Uno de los datos preocupantes, sin duda, es la pobreza existente. El Coneval $^{13}$ refiere que la población con ingreso laboral inferior al costo de la canasta alimentaria (pobreza laboral) aumentó de 38.5\% a 39.3\%, entre el segundo y tercer trimestres de 2018, debido a una disminución en el ingreso laboral, un aumento del costo de la canasta alimentaria y los precios. El poder adquisitivo del ingreso laboral se incrementó desde finales de 2017, pero en el tercer trimestre de 2018 se redujo 1.1\% respecto al trimestre anterior. En el último trimestre, en 26 de las 32 entidades federativas aumentó el porcentaje de población que no puede adquirir la canasta alimentaria con su ingreso laboral. Destacan los estados de Guerrero, Nayarit y Baja California Sur. ${ }^{14}$

Se espera que el Coneval realice una nueva medición de la pobreza en el país para 2019, ya que se lleva acabo cada dos años, y el último informe presentado fue en 2016.

En el sexto informe de gobierno de Enrique Peña Nieto se informó que en su sexenio el padrón del Seguro Popular (el programa social en materia de salud más importante en México) registró un total de 53.3 millones de afiliados con acceso a un catálogo universal de servicios de salud, que cubre 294 intervenciones y 670 medicamentos. ${ }^{15}$ En adición, se logró incorporar un total de 9.2 millones de trabajadores al IMSS, respecto de 2012, ${ }^{16}$ y, por otro lado, se aseguraron a través del seguro facultativo a 6.6. millones de jóvenes estudiantes de educación media superior y superior al seguro de salud. Se redujo en un $9 \%$ la tasa de mortalidad infantil y se celebraron diecisiete semanas nacionales de salud, en las que se aplicaron 163.5 millones de vacunas.

12 Idem.

13 Coneval es acrónimo de Consejo Nacional de Evaluación de la Política de Desarrollo Social, que es una instancia gubernamental mexicana con autonomía y capacidad técnica para generar información objetiva sobre la situación de la política social y la medición de la pobreza en México.

14 Medición de la Pobreza, México, Coneval, 2018, disponible en: https:/ / mww.coneval.org.mx/ Medicion/Paginas/ITLP-IS_resultados_a_nivel_nacional.aspx (fecha de consulta: 23 de enero de 2019).

\footnotetext{
15 Ibidem, p. 53

16 Gobierno de la República, Sexto informe de gobierno, p. 52.
} 
En 2018, las pensiones fueron un tema delicado, ya que las Afores que, como su nombre lo indica, son empresas que se dedican a administrar el dinero de la cuenta individual de los trabajadores, tuvieron pérdidas significativas en el segundo semestre. Ello, debido a que las Afores tienen la facultad para invertir cierto porcentaje de dinero en inversiones como infraestructura, empresas privadas, entre otras, lo que conlleva a la obligación de éstas a buscar el mejor rendimiento para sus afiliados. ${ }^{17}$

Si bien durante el primer trimestre del año las ganancias se mantenían con un incremento de 332,000 millones de pesos (17,446,137 dólares) respecto del primer trimestre de $2017,{ }^{18}$ en la segunda mitad del año se presentó una minusvalía récord de los fondos pensionarios con la cifra histórica de 3 billones 284,428 millones de pesos (172,592,117.7 dólares). Esta pérdida, según la Comisión Nacional del Sistema de Ahorro para el Retiro (Consar), se atribuye, entre otras causas, a la cancelación del "Nuevo Aeropuerto Internacional de México (NAIM)”, ya que Afores como Inbursa, PensionISSSTE, Profuturo y XXI Banorte tenían inversiones en esta obra, que si bien serán indemnizadas, el rendimiento será menor al que se estimaba. ${ }^{19}$

Por su parte, en diciembre, una vez hecho el cambio de gobierno, la Consar mandató que a partir de enero de 2019 se disminuyan las comisiones en $0.03 \%$. que cobran las Afores. ${ }^{20}$

\section{LOS CAMBIOS EN PROGRAMAS Y POLÍTICAS SOCIALES}

Como ya se mencionó, el cambio de los titulares del Ejecutivo y el Legislativo ha traído consigo nuevas políticas y directrices, de tal forma que existen

17 “En qué proyectos está invertido el dinero de las Afores?”, Expansión, México, 2018, disponible en: https://expansion.mx/empresas/2018/04/09/en-que-proyectos-esta-invertido-el-dinerode-las-afores (fecha de consulta: 17 de diciembre de 2018).

18 "Resultados del SAR al primer trimestre del 2018", El Economista, México, 2018, disponible en: https:// wmw.eleconomista.com.mx/opinion/Resultados-del-SAR-al-primer-trimestre-del2018-20180424-0107.html (fecha de consulta: 30 de noviembre de 2018).

19 "Minusvalías por 131,000 mdp en afores, tras cancelación del NAIM", El Economista, en: https:/ / www.eleconomista.com.mx/sectorfinanciero/Minusvalias-por-131000-mdp-en-afores-tras-cancela cion-del-NAIM-20181105-0137.html (fecha de consulta 17 de diciembre de 2018).

20 "Autoriza la Consar disminución de comisiones por manejo de Afores", La Jornada, México, 2018, disponible en: https:/ / wmw.jornada.com.mx/ultimas/2018/12/11/ autoriza-la-consardisminucion-de-comisiones-por-manejo-de-afores-3817.html (fecha de consulta: 14 de diciembre de 2018). 
programas sociales que subsisten en esencia, pero con un nombre diferente o con algunas modificaciones. En ese sentido, los acontecimientos más importantes pueden catalogarse de la siguiente manera:

\section{Programas sociales correspondientes al sexenio 2012-2018}

El Coneval otorgó el reconocimiento "Buenas Prácticas Coneval 2018" al programa Prospera, pues se sostuvo que dicho programa coadyuvó en la reducción de la anemia en niños y niños; en el aumento de utilización de servicios de salud en materia de prevención de enfermedades; el incremento del acceso a la educación, y aumento en el nivel educativo de las familias beneficiadas. ${ }^{21}$

\section{Restructuración de la asistencia social como parte del cambio de gobierno}

La primera transformación se dio a partir del 1o. de diciembre del año en comento, con el cambio de nombre de la Secretaría de Desarrollo Social (Sedesol) ahora Secretaría de Bienestar, la cual, en colaboración con otras secretarías pondrá en marcha los siguientes programas:

Programas de nueva incorporación aprobados en 2018 para su ejercicio en 2019:

- Sembrando vida. Es una estrategia de productividad campesina dirigida a propietarios de tierras ejidales comunales o pequeñas propiedades, con el objetivo de impulsar la siembra de árboles maderables, frutales y milpas intercaladas con árboles frutales; con este programa se pretende generar empleo para cuatrocientos mil productores.

- Precios de garantía a productos alimentarios básicos. Es un programa dependiente de la Secretaría de Agricultura y Desarrollo Rural de ayuda a los pequeños productores agrícolas, en el que se incluyen cuarenta productos a un precio 15\% más bajo que en el mercado, tales como frijol, arroz, pastas, pan, aceite, amaranto, cacahuate, atún, sardina, carne de res, pollo y puerco, huevo y pescado. ${ }^{22}$

21 Programa de Inclusión Social, Prospera es el programa más eficiente que tiene la Administración Pública Federal: Coneval, disponible en: https:// wmw.gob.mx/prospera/prensa/prospera-es-el-programamas-eficiente-que-tiene-la-administracion-publica-federal-coneval (fecha de consulta 16 de enero de 2019).

22 Presenta la Sader nuevos programas con ejercicio presupuestal eficiente y honesto 
- Jóvenes construyendo el futuro. Este programa depende directamente de la Secretaría de Trabajo y Previsión Social y, presupuestalmente, es a la que se le ha destinado mayor recurso; no cuenta con reglas de operación todavía, por lo que se basa en los escuetos lineamientos para la operación del mismo, y es una de las principales líneas de acción del gobierno en turno. Tiene como finalidad capacitar a jóvenes de entre 18 y 29 años que por alguna razón no se encuentren estudiando ni incorporados al mercado laboral, a los cuales se les otorgará una beca mensual durante un año de 3,600.00 pesos. Los becarios tendrán como tarea desarrollar actividades que los capaciten para el trabajo bajo la supervisión de un tutor, tanto en la iniciativa privada, pública, como social; al finalizar el año de capacitación se les otorgará a los becarios una constancia que acredite las habilidades que adquirieron.

Uno de los puntos importantes a destacar es que los becarios serán incorporados al IMSS a través del seguro facultativo, en el cual se contempla la protección contra accidentes, enfermedades, maternidad y riesgos de trabajo. No obstante, no se especifica de dónde se obtendrán los recursos para financiarlo. ${ }^{23}$

Programas que se mantienen.

- Prospera. El programa central del sexenio anterior se mantendrá en 2019, según el presupuesto de egresos aprobado; sin embargo, presenta una disminución en el presupuesto, pues únicamente se le destinarán 15,788,640.00 pesos mexicanos (82,9671.04 dólares), en lugar de $82,729,888.79$ pesos $(4,34,7340.40$ dólares $)$ en $2018 .{ }^{24}$

- Apoyo para migrantes. De la misma forma que Prospera, este programa se mantiene con un presupuesto menor al del año pasado.

- Programa de estancias infantiles para apoyar a madres trabajadoras. Al igual que muchos otros programas, éste no cuenta con reglas de operación para 2019, por lo que si bien se contempla dentro del presupuesto, con una cantidad menor a la aprobada para 2018, se cuestionó en varias discu-

impulsará un sector productivo incluyente, gobierno de México, disponible en: https:// wmw. gob.mx/sader/prensa/presenta-la-sader-nuevos-programas-con-ejercicio-presupuestal-eficiente-y-honestoimpulsara-un-sector-productivo-e-incluyente?idiom =es (fecha de consulta: 24 de enero de 2019).

23 Lineamientos para la operación del programa "Jóvenes Construyendo el Futuro", disponible en: https:/ / wmw.dof.gob.mx / nota_detalle.php?codigo $=5547857$ offecha $=10 / 01 / 2019$ (fecha de consulta: 24 de enero de 2019).

24 Presupuesto de Egresos de la Federación para el ejercicio fiscal 2018. 
siones si estas estancias debían continuar para 2019, de la forma en que habían estado funcionando con anterioridad. ${ }^{25}$

Programas que cambian de nombre.

- Pensiones para el bienestar de las personas con discapacidad permanente: denominado anteriormente como Programa de Atención a Personas con Discapacidad.

- Pensiones para el bienestar de las personas adultas mayores: su nombre anterior a diciembre de 2018 fue Programa de Pensión para Adultos Mayores (65 y más); los cambios más importantes se encuentran en el aumento de presupuesto, pues absorbe el $67 \%$ del presupuesto de la Secretaría de Bienestar. ${ }^{26}$ Con ello se aumenta la edad para las pensiones, que pasa de 65 a 68 años, manteniéndose únicamente en 65 años para las personas indígenas. Durante 2018, el monto asignado fue de 1,160.00 pesos (60.95 dólares) bimestrales y se aprobó aumentarlo a 2,550.00 pesos (133.99 dólares) que se entregarán cada dos meses.

Cabe destacar que, según la administración en turno, se busca hacer un padrón único de beneficiarios de programas sociales, para lo cual, antes de que tomara protesta el actual presidente de la República, en el mes de octubre, veinte mil 27"voluntarios" 28 denominados "servidores de la nación" realizaron el "censo del bienestar" con el objetivo de recabar información de la problemática ante la que se enfrentan los hogares en México. Lo anterior, pese a existir el Instituto Nacional de Estadística y Geografía, que cuenta con la infraestructura y capacitación para levantar los censos oficiales en el país.

25 Véase, con punto de acuerdo, por el cual se exhorta a la Sedesol a establecer mesas de diálogo con los representantes de las estancias infantiles en cada una de las 32 entidades federativas para considerar sus demandas y propuestas en la definición de las reglas de operación del programa de estancias infantiles de 2019, suscrita por los diputados Claudia Angélica Domínguez Vázquez, Óscar González Yáñez y José Luis Montalvo Luna, del grupo parlamentario del PT, disponible en: http:/ / sil.gobernacion.gob.mx/Archivos/Documentos/2018/10/ asun_3746721_20181004_1538663164.pdf(fecha de consulta: 24 de enero de 2019).

26 "Presupuesto para apoyo pensionario de adultos mayores crece 148\%", El Economista, disponible en: https:// www.eleconomista.com.mx/sectorfinanciero/Presupuesto-para-apoyo-pensionario-deadultos-mayores-crece-148-20181226-0062.html, fecha de consulta 23 de enero de 2019.

27 "Veinte mil «servidores de la nación» aplican el Censo para el bienestar en todo el país", La Jornada, disponible en: https://www.jornada.com.mx/2018/10/06/politica/009n1pol (fecha de consulta: 23 de enero de 2019).

28 Contaron con un apoyo de 3,500.00 pesos mexicanos. 


\section{Vivienda}

En septiembre de 2017 se registraron dos sismos de alta intensidad, cuyas consecuencias debieron ser atendidas durante todo 2018. El primer sismo fue el 7 de septiembre, con una magnitud 8.2, y afectó los estados de Chiapas, Veracruz y Oaxaca; ${ }^{29}$ el segundo fue el 19 de septiembre, de magnitud 7.1 en la escala de Richter, y afectó gravemente los estados de Morelos, Puebla, Tlaxcala, Ciudad de México, Oaxaca, Chiapas, Veracruz y Guerrero. ${ }^{30}$ Se estima que doce millones de personas fueron afectadas, y en total, por los dos sismos se registraron dieciséis mil planteles educativos dañados, 53 centros de salud con daños mayores, y mil 821 edificaciones culturales con daños. ${ }^{31}$

Además, 180 mil 731 viviendas se reportaron con daños; más de 250 mil mexicanos se quedaron sin su vivienda, y hoy están en situación de pobreza patrimonial. ${ }^{32}$

En ese sentido, es importante analizar de manera concisa el papel que ha desempeñado la seguridad social en 2018, en atención a la protección encaminada a la vivienda.

En México, los principales institutos del seguro social a nivel nacional encargados de brindar créditos para la vivienda son: el Instituto del Fondo Nacional de la Vivienda para los Trabajadores (Infonavit) y el Fondo de la Vivienda del Instituto de Seguridad y Servicios Sociales de los Trabajadores del Estado (Fovissste).

El Infonavit publicó diez programas de ayuda para los derechohabientes afectados por los sismos.

29 Reporte especial, Servicio Sismológico Nacional, UNAM, disponible en: http:// wmm.ssn. unam.mx/sismicidad/reportes-especiales/2017/SSNMX_rep_esp_20170907_Tebuantepec_M82.pdf (fecha de consulta: 12 de agosto de 2018).

30 Reporte especial, Servicio Sismológico Nacional, UNAM, disponible en: http:// wmw.ssn. unam.mx/sismicidad/reportes-especiales/2017/SSNMX_rep_esp_20170919_Puebla-Morelos_M71.pdf (fecha de consulta: 13 de agosto de 2018).

31 Plan de acción ante sismos 7 y 8 de septiembre de 2017, disponible en: https:/ / es.scribd. com/document/361993553/Plan-de-Accion-Ante-Sismos-Sep-2017-171017 (fecha de consulta: 12 de agosto de 2018).

32 "Presenta hoy AMLO en Jojutla el Plan Nacional de Reconstrucción", La Jornada, disponible en: https://mwm.jornada.com.mx/ultimas/2018/12/11/presenta-boy-amlo-en-jojutla-el-plannacional-de-reconstruccion-8485.html (fecha de consulta 12 de agosto de 2018). 
En el último informe del Infonavit de agosto de 2018, sobre los créditos otorgados reportó haber atendido a 6,060 personas por concepto de movilidad habitacional. ${ }^{33}$

Por su parte, el Fovissste hasta principios de octubre destinó 114.5 millones de pesos y atendió a 6,851 acreditados que sufrieron afectaciones totales o parciales derivadas de los sismos. ${ }^{34}$

Independientemente de lo realizado por los institutos mencionados con anterioridad, existe un Fideicomiso Fondo de Desastres Naturales (Fonden), destinado a apoyar a las entidades federativas que sufran algún daño derivado de los desastres naturales, en la atención y recuperación de los efectos que estos provoquen. ${ }^{35}$

Este Fideicomiso, a diferencia de los otros dos enlistados con anterioridad, no depende de la relación laboral que tuviera el afectado, pues los apoyos se otorgan tanto a personas como a instituciones. El monto destinado para la reconstrucción se divide en apoyos parciales inmediatos, con una cantidad de 6 mil 844 millones de pesos (359,642,669 dólares), y de apoyos para la reconstrucción 22 mil 810 millones 342 mil 755 pesos (1,198,651,747 dólares), de los cuales menos de la mitad se destinó a la reconstrucción de vivienda. ${ }^{36}$

\section{PRINCIPALES RESOluCIONES DE LA SUPREMA CORTE DE JUSTICIA DE LA NACIÓN}

Desde hace algunos años, las resoluciones de la SCJN han tomado seriamente en consideración el análisis de los actos reclamados a partir de la vulneración de alguno de sus derechos humanos. Bajo esa tesitura, si bien por cantidad las tesis no son muchas, la Corte ha emitido criterios importantes en materia de seguridad social, tales como:

33 Total de acciones de crédito, Infonavit, disponible en: http://portal.infonavit.org.mx/mps/ wcm/connect/27cd44d4-d403-4f1a-83a3-645aff70746/Movilidad_habitacional_medidas_emergentes_ desastres.pdf?MOD=AJPERES\&CONVERT_TO=url\&CACHEID=ROOTWORKSPACE27cd44d4-d403-4f1a-83a3-b45a0ff70746-mkqCowt (fecha de consulta: 13 de agosto de 2018).

34 "Fovissste atiende a más de seis mil derechos habientes afectados por los sismos", El Universal, disponible en: http:// wmw.eluniversal.com.mx/cartera/ economia/ fovissste-atiende-mas-de-seismil-derechobabientes-afectados-por-sismos (fecha de consulta: 13 de agosto de 2018).

35 Fonden, Secretaría de Gobierno, disponible en: https://wmm.gob.mx/segob/documentos/ fideicomiso-fondo-de-desastres-naturales-fonden (fecha de consulta: 13 de agosto de 2018).

36 Fonden, Fuerza México, transparencia, disponible en: http:/ / wmw.transparenciapresupuestaria. gob.mx/es/PTP/fuerzamexico (fecha de consulta: 16 de agosto de 2018). 


\section{Pensión por ascendencia}

Pensión por ascendencia. El artículo 75, fracción V, de la Ley del Instituto de Seguridad y Servicios Sociales de los Trabajadores del Estado, vigente hasta el 31 de marzo de 2007, al establecer como requisito para su otorgamiento que se acredite la dependencia económica con el trabajador o pensionista durante los cinco años anteriores a su muerte, es inconstitucional. ${ }^{37}$

Para acceder a una pensión por ascendencia en el Instituto de Seguridad y Servicios Sociales de los Trabajadores del Estado (ISSSTE) se deben satisfacer determinados requisitos, los cuales se contemplan en el artículo 75 de la Ley del instituto. Uno de ellos es acreditar la dependencia económica durante los cinco años anteriores a la muerte del trabajador o pensionista, cuestión que llevó a conformar un nuevo criterio a raíz del siguiente caso:

La madre de un trabajador fallecido interpuso un juicio de amparo contra una sentencia emitida por un tribunal en materia administrativa que había negado el derecho de percibir la pensión del trabajador fallecido, por no poder acreditar la dependencia económica de cinco años requeridos en el artículo antes señalado.

Después de haberse agotado todo el procedimiento correspondiente, la sentencia de amparo le fue concedida a la solicitante, pues el órgano jurisdiccional, al resolver el asunto, consideró la situación de vulnerabilidad en la que se encontraba la madre del trabajador fallecido, pues tenía 86 años, por lo cual dejaba claro la complicación de poder obtener recursos para su subsistencia, advirtiendo la dependencia que tenía de su hijo. A su vez, se hace mención sobre la ausencia de consideración de lo que establece el Código Civil Federal, donde se regula que los hijos están obligados a dar alimentos a los padres. ${ }^{38}$

Este amparo es una muestra del activismo judicial que se inicia en México, que permite que la interpretación de la ley sea más amplia y acorde a los derechos humanos, no solamente a los requisitos establecidos en las normas o la presunción de la prueba; con esta interpretación se hace el reconocimiento de un derecho humano de subsistencia para una persona mayor.

37 Tesis: (V Región) 4o.1 A (10a.), Gaceta del Semanario Judicial de la Federación, Décima Época, libro 52, t. IV, marzo de 2018, p. 3432.

38 Idem. 


\section{Reparación del daño a beneficiarios de policía}

Seguridad social para los beneficiarios de los elementos policiacos fallecidos en servicio. El derecho de aquéllos a gozar de las prestaciones relativas que otorga el Instituto de Seguridad y Servicios Sociales de los Trabajadores del Estado no está supeditado a que la entidad pública en la que éstos se desempeñaban celebre el convenio respectivo con dicho organismo, por lo cual, debe inscribirlos al régimen obligatorio cuando lo soliciten. ${ }^{39}$ Tesis: XI.3o.A.T.6 A (10a.), marzo de 2017, Décima Época, Registro: 2018092, Tribunales Colegiados de Circuito.

Los policías mexicanos, en materia de seguridad social, se encuentran en una gran incertidumbre de aseguramiento, ya que por la naturaleza de su trabajo dependen del Poder Ejecutivo federal, estatal y local. En ese sentido, ellos pueden ser asegurados, ya sea en el ISSSTE, celebrar convenios con el IMSS o bien tener atención médica a través de seguros privados. Dicho lo anterior, existen casos en donde los municipios no aseguran a sus miembros policiacos en ninguno de los tres supuestos anteriores, con lo que vulneran su derecho humano a la seguridad social.

En ese tenor, un tribunal en materia administrativa y de trabajo generó una tesis derivada de un amparo indirecto interpuesto por la viuda de un policía que murió por un disparo mientras se trasladaba a su domicilio y aún portaba su uniforme. El acto reclamado consiste en la violación de los derechos humanos fundamentales reconocidos en nuestra carta magna, como el de la salud y seguridad social, contenidos en la Convención Americana de Derechos Humanos, los cuales fueron vulnerados por la autoridad municipal, ya que negó a la viuda su aseguramiento ante el ISSSTE.

El tribunal colegiado resolvió que, efectivamente, el trabajador fallecido y su familia sufrieron perjuicios por no haberlos asegurado ante el ISSSTE para que aquélla pudiera tener acceso a las prestaciones de seguridad social correspondientes y condenó a la autoridad responsable a lo siguiente:

- Derecho al pago de gastos funerarios.

- Derecho al pago de cinco mil días de salario por concepto de indemnización.

- Derecho al pago de un seguro de vida.

39 Tesis: XI.3o.A.T.6 A (10a.), marzo de 2017, Gaceta del Seminario Judicial de la Federación, Décima Época, libro 59, t. III, octubre de 2008, p. 2492. 
- Pago de tres meses de salario por concepto de indemnización.

- Daños y perjuicios que se desprendan, entre ellas las aportaciones de cuotas al sistema de ahorro para el retiro. ${ }^{40}$

Esta resolución marca un precedente sumamente importante en el país para la justicia en materia de seguridad social para los trabajadores policiacos y sus familias.

\section{Seguridad social para trabajadores domésticos}

En México, el acceso de los trabajadores domésticos al Instituto Mexicano del Seguro Social (IMSS) se contempla en la llamada, ilógicamente, "incorporación voluntaria al régimen obligatorio", es decir, queda al arbitrio del empleador si los asegura o no; sin embargo, la Suprema Corte de Justicia de la Unión emitió una trascendente resolución en la materia; ello, derivado del siguiente caso:

En abril de 2016, una empleada doméstica presentó una demanda ante la Junta Local de Conciliación y Arbitraje de la Ciudad de México, en la cual demandó las prestaciones de indemnización constitucional, pago de salarios caídos, aguinaldo, vacaciones, prima vacacional, prima de antigüedad y del tiempo extraordinario, así como la inscripción retroactiva ante el Instituto Mexicano del Seguro Social. La Junta resolvió que se le tenían que pagar las prestaciones demandadas, a excepción de la indemnización constitucional, y, además, absolvió al demandado por la inscripción retroactiva ante el IMSS de su extrabajadora. Por tal motivo, la trabajadora, acorde a su inconformidad, promovió un juicio de amparo por estimar vulnerados sus derechos humanos, al considerar que el artículo 13 de la Ley del Seguro Social es inconstitucional (no obliga a los patrones a asegurar a sus trabajadores domésticos).

Derivado de la importancia del asunto, la Suprema Corte de Justicia de la Nación atrajo el caso y resolvió dar una ampliación a la cobertura de seguridad social, tomando como base los derechos humanos, de la siguiente manera:

- El hecho de que los patrones no tengan la obligación jurídica de inscribir a los trabajadores domésticos ante el IMSS constituye un trato dis-

40 Idem. 
criminatorio por el artículo 1o. constitucional, así como una violación al derecho humano a la seguridad social.

- Debe tomarse en cuenta el principio de igualdad para el acceso a la seguridad social de los trabajadores domésticos.

- No existe una razón válida, constitucionalmente, para que la Ley Federal del Trabajo y del IMSS excluyan al trabajo doméstico del régimen obligatorio de seguridad social

- El Estado debe tomar medidas, hasta el máximo de los recursos que disponga, "para que los sistemas de seguridad social incluyan a los trabajadores insuficientemente protegidos por la seguridad social".

- Reconoce que "la importancia fundamental de la seguridad social para la dignidad humana y el reconocimiento jurídico de este derecho por los Estados Partes supone que se le debe dar la prioridad adecuada en la legislación y en la política del Estado", y, por tanto, el Estado deberá adoptar las medidas necesarias para que toda persona disfrute del derecho a la seguridad social lo antes posible, entendiendo que el Estado puede generar regímenes de seguridad social diferenciados para atender las distintas necesidades de la población, incluidos los grupos vulnerables o marginados.

- Resalta la importancia de la seguridad social como un actor que desempeña un papel importante para reducir y mitigar la pobreza, prevenir la exclusión social y promover la inclusión social.

- Recomienda al IMSS crear un programa piloto, en un plazo no mayor de dieciocho meses, y que proponga al Congreso de la Unión las adecuaciones legales para la incorporación de los trabajadores domésticos a un nuevo sistema especial de seguridad social, en un plazo no mayor de tres años.

- El nuevo esquema de aseguramiento no puede ser de carácter voluntario, sino imperativo, y debe resultar de fácil implementación para los patrones. $^{41}$

\section{REFORMAS LEGISLATIVAS EN 2018}

Durante 2018 se aprobaron diversas reformas legislativas que impactan directamente en temas relacionados con la seguridad social, tales como:

41 Sentencia de amparo directo 9/2018 resuelto por la Segunda Sala de la Suprema Corte de Justicia de la Nación a cargo del ministro ponente Alberto Pérez Dayán, disponible en: bttps:/ / www.scjn.gob.mx/sites/default/files/listas/documento_dos/2018-11/ A.D.\%209-2018.pdf (fecha de consulta: 6 de febrero de 2019). 


\section{Riesgos laborales}

El 22 de junio de 2018 se publicó en el DOF la Ley Federal de Declaración Especial de Ausencia para Personas Desaparecidas, mediante decreto presidencial, a diferencia del procedimiento establecido por el Código Civil Federal, que establece que la declaración de ausencia se podrá solicitar pasados dos años después del aviso al Ministerio Público de su desaparición. Hoy en día, con la promulgación de esta Ley, el procedimiento podrá solicitarse a partir de los tres meses en que se haya hecho la denuncia de desaparición o la presentación de queja ante la Comisión Nacional de los Derechos Humanos. ${ }^{42}$ Lo trascendente en materia de derechos sociales es el artículo 21, fracción VI, que establece que la declaración especial de ausencia tendrá — entre otros - como mínimo el efecto de "Permitir que las personas beneficiarias de un régimen de seguridad social derivado de una relación de trabajo de la Persona Desaparecida continúen gozando de todos los derechos y beneficios aplicables a este régimen", ${ }^{43}$ y para ello, se reforman las siguientes leyes:

- Ley Federal del Trabajo. Se adiciona la fracción XXIX al artículo 132, que establece como obligación del patrón, otorgar permiso sin goce de sueldo a las y los trabajadores declarados desaparecidos que cuenten con declaración especial de ausencia. En ese mismo sentido, en la fracción XVI del artículo 133 impone la prohibición al patrón, de dar de baja o terminar la relación laboral de un trabajador que tenga la calidad de persona desaparecida y cuente con declaración especial de ausencia. $^{44}$

- Ley del Seguro Social. El artículo 109 bis establece que cuando el trabajador tenga la calidad de persona desaparecida y cuente con declaración

42 Ley Federal de Declaración Especial de Ausencia para Personas Desaparecidas, artículo 8.

43 Diario Oficial de la Federación, Decreto por el que se expide la Ley Federal de Declaración Especial de Ausencia para Personas Desaparecidas, y se reforman diversas disposiciones de la Ley Federal del Trabajo; de la Ley Federal de los Trabajadores al Servicio del Estado, Reglamentaria del Apartado B) del Artículo 123 Constitucional; de la Ley del Seguro Social; de la Ley del Instituto de Seguridad y Servicios Sociales de los Trabajadores del Estado; de la Ley General de Títulos y Operaciones de Crédito; de la Ley de Instituciones de Crédito y de la Ley Agraria, disponible en: http:// wmw.dof.gob.mx/nota_detalle.php?codigo=5528936 \&.fec $h a=22 / 06 / 2018$ (fecha de consulta: 31 de julio de 2018).

44 Ley Federal de Trabajo, Diario Oficial de la Federación, disponible en: http:/ / wmw.diputados. gob.mx/LeyesBiblio/pdf/125_220618.pdf(fecha de consulta: 31 de julio de 2018). 
especial de ausencia, los beneficiarios conservarán el derecho a recibir la asistencia médica y de maternidad, quirúrgica, farmacéutica y hospitalaria que sea necesaria.

- Ley del ISSSTE adiciona como riesgo de trabajo la desaparición derivada de un acto delincuencial en el artículo 56, fracción V. Además de ello, se modifican los artículos 66 y 67, en los que se posibilita que los beneficiarios de los trabajadores desaparecidos a consecuencia de un acto delincuencial obtengan las prestaciones en especie (atención médica, quirúrgica, hospitalaria y farmacéutica) y económicas (pensiones) que se otorgan en caso de riesgo de trabajo.

\section{Pensiones}

El 5 de noviembre de 2018, por orden del presidente de la Cámara de Senadores, se publicó en el Diario Oficial de la Federación la Ley Federal de Remuneraciones de los Servidores Públicos, reglamentaria de los artículos 75 y 127 de la Constitución Política de los Estados Unidos Mexicanos, con la cual se pone fin al otorgamiento de pensiones a los ex titulares del Poder Ejecutivo federal. Cabe aclarar que la Ley Federal de Remuneraciones de los Servidores Públicos no es una ley exclusivamente encaminada a eliminar dichas pensiones, sino que dentro de los artículos 10 y 11 se establece que únicamente se concederán pensiones, jubilaciones, compensaciones o cualquier otra prestación de naturaleza semejante cuando éstas sean asignadas expresamente por una ley, decreto legislativo, o bien si se encuentren señaladas dentro del contrato colectivo o condiciones generales de trabajo. En ese tenor, no podrán concederse por el simple acuerdo de los titulares de los entes públicos ni de sus órganos de gobierno.

Bajo ese contexto, el artículo transitorio segundo sostiene que una vez entrada en vigor dicha ley quedarán sin efectos todas las disposiciones contrarias a la misma, dentro de las cuales se encuadran las pensiones de los expresidentes que fueron otorgadas a través de un acuerdo presidencial.

\section{Atención a la salud}

En diciembre de 2018, a través del denominado "Acuerdo para Garantizar el Derecho a la Salud", el actual presidente de la República, Andrés Manuel López Obrador, presentó este mandato, que incide en los estados de Campe- 
che, Chiapas, Guerrero, Oaxaca, Quintana Roo, Tabasco, Veracruz y Yucatán, en donde se contempla dar acceso universal a la salud a todas las personas. Cabe destacar que este acuerdo es la primera fase del proyecto de creación del Sistema Nacional de Salud para el Bienestar, que busca incorporar a todas las entidades federativas del país en un plazo de dos años. ${ }^{45}$

El primer nivel de atención se cubrirá en las clínicas, centros de salud y unidades médicas del IMSS-Bienestar, y uno de los objetivos finales es que todas las instituciones de seguridad social colaboren en la atención de la población no derechohabiente.

Es importante destacar que lo que busca este proyecto es sustituir al llamado "seguro popular"; sin embargo, ambos programas tienen el mismo objetivo principal: brindar atención a la salud a la población no asegurada. Si bien el seguro popular intentó dar cumplimiento a diversos mandatos internacionales, como la Declaración de Copenhague sobre Desarrollo Social y el Programa de Acción de la Cumbre Mundial, los Objetivos de Desarrollo del Milenio de 2000 y la Agenda 2030 para el Desarrollo Sostenible, el principal cambio no está en el objetivo, sino en la institución que será responsable. Mientras que el seguro popular de salud se desarrolló a través de un sistema de salud gestionado directamente por la Secretaría de Salud, la administración del Sistema de Salud para el Bienestar será a través del IMSS. Es decir, un seguro social creado para los trabajadores de la iniciativa privada será el responsable de dar cobertura en salud a la población no derechohabiente.

Esto representa grandes incógnitas; por ejemplo, determinar la legislación, la cobertura, el financiamiento, etcétera.

Una interrogante grande es la cobertura en las prestaciones que tendrá, porque mientras el seguro popular determina hoy en día qué prestaciones médicas otorga a través de un catálogo universal de servicios de salud que cubre 294 intervenciones y 670 medicamentos, el IMSS da cobertura de atención universal determinando en su reglamentación un pequeño grupo de prestaciones que no otorga; por ejemplo, el artículo 42 del Reglamento de Prestaciones Médicas del Instituto Mexicano del Seguro Social señala que no se otorgarán anteojos o prótesis dentales.

45 Acuerdo para garantizar el \#DerechoALaSalud, México, Gobierno de la República, 2018, disponible en: https://mmm.gob.mx/presidencia/articulos/acuerdo-para-garantizar-el-derechoala salud?idiom $=e s$ (fecha de consulta: 27 de enero de 2019). 


\section{EXPECTATIVAS DE LEY}

\section{Licencia para padres con hijos enfermos de cáncer}

Ambas Cámaras del Congreso de la Unión han aprobado adiciones a diversas disposiciones de la Ley Federal del Trabajo, de la Ley del Seguro Social y de la Ley del Instituto de Seguridad y Servicios Sociales de los Trabajadores del Estado, en el que se otorgue una licencia a madres y padres trabajadores con hijos menores de dieciséis años diagnosticados con cáncer, en caso de que el menor requiera descanso médico u hospitalización. ${ }^{46}$

La licencia tiene una duración de uno hasta veintiocho días, y puede expedirse cuantas licencias sean necesarias en un periodo máximo tres años, sin que éstas excedan 364 días, los cuales no necesariamente deberán ser continuos.

Esta reforma contempla la adición de varios conceptos importantes que benefician la conciliación de la vida laboral y familiar. ${ }^{47}$

\section{Iniciativa de reforma laboral}

Presentada por el grupo parlamentario de Morena, contempla diversos cambios a la Ley Federal de Trabajo, siendo el de mayor trascendencia en materia sindical, de transparencia, rendición de cuentas, creación de centros

46 Uno de los motivos que dieron origen a esta iniciativa es que el cáncer infantil es la causa número uno de muerte entre niños de cinco a catorce años de edad en México, de acuerdo con cifras de la Secretaría de Salud federal. Asimismo, actualmente hay más de 23,000 familias que tienen un caso de cáncer infantil en sus hogares, y cada año aumentan en 5,000 el número de diagnosticados con esta enfermedad. Considerando que un menor diagnosticado con cáncer que se encuentra en un tratamiento oncológico requiere (por protocolo médico) del acompañamiento de alguno de sus padres o tutores; sin embargo, dicha situación compromete la estabilidad en el empleo de sus padres o tutores (al ausentarse del empleo para acompañarlos), por lo que padres o tutores dejan de tener la posibilidad de acceder a un empleo digno establecido en el artículo 5o. de la CPEUM. Resulta claro que se requieren cambios a la legislación secundaria, a fin de que el ejercicio de la garantía del interés superior de la niñez no impida o comprometa el acceso a un empleo digno. Cámara de Diputados, Iniciativas, disponible en: http:// sitl.diputados.gob.mx/LXIV_leg/iniciativaslxiv.php?comt=47ङ turnot=1 \&edot $=T$ (fecha de consulta: 10 de enero de 2019).

47 Dictamen de las comisiones unidas de trabajo y previsión social de estudios legislativos, Senado de la República, Proyecto de Decreto por el que se adicionan diversas disposiciones de la LFT, LSS, Ley del ISSSTE, 2018. 
de conciliación, así como la implementación de tribunales laborales. Estas acciones supondrían el fin de la justicia laboral como se conoce hoy en día, en donde los asuntos se resuelven por un órgano colegiado avalado por un representante patronal, uno de los trabajadores y uno del Estado.

\section{Seguridad social para parejas del mismo sexo en el IMSS e ISSSTE}

En años anteriores ha habido avances en materia jurisprudencial sobre la incorporación a los regímenes de seguridad social, así como de pensiones que tenían las personas del mismo sexo. Si bien en otros países esto es un tema superado, en México su discusión aún prevalece; tan es así, que el 7 de noviembre de 2018 el pleno de senadores aprobaron por unanimidad de 110 votos las reformas a las leyes del IMSS y del ISSSTE para que se elimine el lenguaje sexista y discriminatorio en dichas normas. La propuesta, básicamente consiste en reconocer la protección social de un derechohabiente a su beneficiario, esposo, esposa o cónyuge del mismo sexo.

Aún no es posible hablar de una reforma integral en materia de seguridad social, puesto que dicha minuta fue enviada a la Cámara de Diputados para su revisión. ${ }^{48}$

\section{REFLEXIONES A MANERA DE CONCLUSIÓN}

Los retos ante los que se enfrenta la seguridad social en México implican un constante cambio de estrategia para poder enfrentarlos, pues, como se analizó anteriormente, en específico, 2018 fue un año de grandes contrastes y pocas reformas legislativas.

Por una parte, los criterios emitidos por la SCJN fueron por demás trascendentes en la atención de la seguridad social para dos grupos que a lo largo de la historia han visto vulnerados sus derechos en la materia: trabajadores domésticos y policías. Pese a ello, aún queda pendiente la positivización de estos derechos, no sólo para aquellos que recurran las sentencias que no les han sido favorables, sino para toda la población que se encuentre en un estado de vulnerabilidad.

48 Arvizu, Juan, Avala Senado seguridad social a parejas del mismo sexo, disponible en: http:// www.eluniversal.com. $m x$ /nacion/ avala-senado-seguridad-social-parejas-del-mismo-sexo (fecha de consulta: 20 de noviembre de 2018). 
Por otro lado, en el área referente a los programas sociales la expectativa sembrada en la campaña presidencial de Andrés Manuel López Obrador es grande. Seguramente durante 2019 se empezarán a ver los resultados de este conjunto de cambios realizados en el presupuesto y cambio de dirección en los programas sociales, en el cual todos esperamos que finalmente reduzcan la pobreza. No se puede omitir señalar que también esperamos que dichos cambios vayan acompañados de la legislación necesaria para dar soporte jurídico a la operación de los programas y certeza jurídica a los beneficiarios de los mismos.

\section{FUENTES DE INVESTIGACIÓN}

Arvizu, Juan, Avala Senado seguridad social a parejas del mismo sexo, disponible en: http:// wmw.eluniversal.com.mx/nacion/avala-senado-seguridad-social-parejasdel-mismo-sexo.

"Autoriza la Consar disminución de comisiones por manejo de Afores", La Jornada, México, 2018, disponible en: https://mww.jornada.com.mx/ultimas /2018/12/11/ autoriza-la-consar-disminucion-de-comisiones-por-manejo-de-afores-3817.html

Ayuntamiento de Xalapa, Caravana migrante, México, 2018, disponible

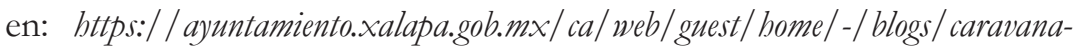
migrante / maximized? refererPlid $=20137$.

BANCO DE MÉXICO, Inflación, disponible en: http://mww.anterior.banxico.org. $m x /$ portal-inflacion/inflacion. btml.

BBVA RESEARCH, Remesas imparables, bilan 30 meses con crecimiento, $+8.8 \%$ en septiembre, México, 2018, disponible en: https:/ / wnw.bbvaresearch.com/wp-con tent/uploads/2018/11/20181101_FlashMigracion.pdf.

"Cámara de Diputados aprueba licencias de hasta 3 años para trabajadores con hijos con cáncer", Reporte Índigo, disponible en: https:/ / www.reporteindigo.com/ reporte / camara-de-diputados-aprueba-licencias-de-hasta-3-anos-para-trabajadores-con-hijos-con-cancer/

CÁMARA DE DipuTADOs, Iniciativas, disponible en: http:/ / sitl.diputados.gob.mx/ LXIV_leg/iniciativaslxiv.php?comt $=47$ \&tipo_turnot $=1$ \&edot $=T$.

CNN EsPañol, México, el país donde hay más de 32 mil desaparecidos, disponible en: https:// cnnespanol.cnn.com/2017/09/13/mexico-el-pais-donde-hay-mas-de32-000-desaparecidos/. 
Coneval, Medición de la pobreza, México, 2018, disponible en: https://wmw. coneval.org.mx/Medicion/Paginas/ITLP-IS_resultados_a_nivel_nacional.aspx.

CONGRESO DE LA UNIÓN, Iniciativa que reforma, adiciona y deroga diversas disposiciones de la Ley Federal del Trabajo, Grupo Parlamentario de Morena, disponible en: http://gaceta.diputados.gob.mx/Gaceta/64/2019/ene/20190103-II. btml\#Iniciativa2.

“¿En qué proyectos está invertido el dinero de las Afores?”, Expansión, México, 2018, disponible en: https://expansion.mx/empresas/2018/04/09/enque-proyectos-esta-invertido-el-dinero-de-las-afores.

FONDEN, Fuerza México, transparencia, disponible en: http:/ / www.transparenciapresupuestaria.gob.mx/es/PTP/fuerzamexico.

"Fovissste atiende a más de seis mil derechos habientes afectados por los sismos", El Universal, México, disponible en: http:// wmw.eluniversal.com. $m x /$ cartera/ economia/fovissste-atiende-mas-de-seis-mil-derechobabientes-afectadospor-sismos.

FueRZA MÉXICO, Los pasos que se siguieron para la atención de los sismos del 7 y 19 de septiembre, disponible en: https:/ / www.transparenciapresupuestaria.gob.mx/ es/PTP/fuerzamexico

GOB. Acuerdo para garantizar el \#Derecho ALaSalud, disponible en: https: / / www. gob.mx/presidencia/articulos/acuerdo-para-garantizar-el-derechoalasalud?idiom $=e s$

GOBIERNO DE MÉXICO, Jóvenes construyendo el futuro, México, 2018, en: https:/ / jovenesconstruyendoelfuturo.stps.gob.mx/.

GOBIERNO DE MÉXICO, Presenta la SADER nuevos programas con ejecicio presupuestal eficiente y honesto impulsará un sector productivo incluyente, disponible en: https://wmm.gob.mx/sader/prensa/presenta-la-sader-nue vos-programas-con-ejercicio-presupuestal-eficiente-y-honesto-impulsara-un-sector-pro ductivo-e-incluyente? idiom $=e$.

IMSS, IMSS Bienestar para todos, Gobierno de México, 2018.

INE, "López Obrador gana con 30 millones de votos", Proceso, disponible en: https:/ / www.proceso.com.mx/541748/ ine-lopez-obrador-gana-con-30-millones-devotos-y-el-53-17-por-ciento.

INEGI, Resultados de la Encuesta Nacional de Ocupación y Empleo, México, 2018, disponible en: http://www.beta.inegi.org. $m x$ / app/saladeprensa/noticia. btml?id $=4558$.

INEGI, Glosario, disponible en http:/ / mmw.beta.inegi.org.mx/app/glosario/default. html? $p=E N E U$ 
INFONAVIT, Total de acciones de crédito, disponible en: http:/ /portal.infonavit.org.mx/ wps / wcm/connect/27cd44d4-d403-4f1a-83a3-b45a0ff70746/Movilidad_habitacional_medidas_emergentes_desastres.pdf?MOD $=$ AJPERES $\odot \cdot C O N V E R T$ TO= urle 2 CACHEID $=$ ROOTWORKSPACE-27cd44d4t-d403-4f1 a-83a3b45aff70746-mkqCowt.

Ley Federal de Trabajo, Diario Oficial de la Federación, disponible en: http:/ / mmn. diputados.gob.mx/LeyesBiblio/pdf/125_220618.pdf.

"Migrantes en asamblea rechazan el plan de Peña Nieto", El Universal, México, disponible en: https:// www.eluniversal.com.mx/ nacion/politica/ migrantesen-asamblea-rechazan-plan-de-pena-nieto.

"Minusvalías por 131,000 mdp en afores, tras cancelación del NAIM", El Economista, México, disponible en: https://www.eleconomista.com.mx/ sectorfinanciero/Minusvalias-por-131000-mdp-en-afores-tras-cancelacion-delNAIM-20181105-0137.btml.

"Presenta hoy AMLO en Jojutla el Plan Nacional de Reconstrucción”, La Jornada, México, disponible en: https://mmm.jornada.com.mx/ultimas/2018/12/11/ presenta-hoy-amlo-en-jojutla-el-plan-nacional-de-reconstruccion-8485.html.

"Presupuesto para apoyo pensionario de adultos mayores crece 148\%", El Economista, México, disponible en: https://mwn.eleconomista.com.mx/sectorfinanciero/Presupuesto-para-apoyo-pensionario-de-adultos-mayores-crece-1482018 1226-0062. html

Programa de Inclusión Social, Prospera es el programa más eficiente que tiene la Administración Pública Federal, Coneval, disponible en: https:/ / wmm.gob.mx/ prospera/prensal prospera-es-el-programa-mas-eficiente-que-tiene-la-administracionpublica-federal-coneval.

"Resultados del SAR al primer trimestre del 2018", El Economista, México, 2018, disponible en: https:/ / wnw.eleconomista.com.mx/ opinion/Resultados-delSAR-al-primer-trimestre-del-2018-20180424-0107.html.

RNPED, Acciones y programas, disponible en: bttps:// wmm.gob.mx/sesnsp/accionesy-programas/registro-nacional-de-datos-de-personas-extraviadas-o-desaparecidas-rnped.

SENAdO DE la RepúbliCA, Dictamen de las comisiones unidas de trabajo y previsión social de estudios legislativos. Proyecto de Decreto por el que se adicionan diversas disposiciones de la LFT, LSS, Ley del ISSSTE, 2018.

SERVICIO Sismológico NACiOnAL, Reporte especial, UNAM, disponible en: http:/ / www.ssn.unam.mx/sismicidad/ reportes-especiales/2017/SSNMX_rep_ esp_20170907_Tehuantepec_M82.pdf. 